\title{
The Particle Problem in the General Theory of Relativity
}

\author{
A. Einstein And N. Rosen, Institute for Advanced Study, Princeton
}

(Received May 8, 1935)

\begin{abstract}
The writers investigate the possibility of an atomistic theory of matter and electricity which, while excluding singularities of the field, makes use of no other variables than the $g_{\mu \nu}$ of the general relativity theory and the $\varphi_{\mu}$ of the Maxwell theory. By the consideration of a simple example they are led to modify slightly the gravitational equations which then admit regular solutions for the static spherically symmetric case. These solutions involve the mathematical representation of physical space by a space of two identical sheets, a particle being represented by a "bridge" connecting these sheets. One is able to understand why no neutral particles of negative mass are to be
\end{abstract}

found. The combined system of gravitational and electromagnetic equations are treated similarly and lead to a similar interpretation. The most natural elementary charged particle is found to be one of zero mass. The manyparticle system is expected to be represented by a regular solution of the field equations corresponding to a space of two identical sheets joined by many bridges. In this case, because of the absence of singularities, the field equations determine both the field and the motion of the particles. The many-particle problem, which would clecide the value of the theory, has not yet been treated.

One would be inclined to answer this question in the negative in view of the fact that the Schwarzschild solution for the spherically symmetric static gravitational field and Reissner's extension of this solution to the case when an electrostatic field is also present each have a singularity. Furthermore the last of the Maxwell equations, which expresses the vanishing of the divergence of the (contravariant) electrical field density, appears to exclude in general the existence of charge densities, hence also of electrical particles.

For these reasons writers have occasionally noted the possibility that material particles might be considered as singularities of the field. This point of view, however, we cannot accept at all. For a singularity brings so much arbitrariness into the theory that it actually nullifies its laws. A pretty confirmation of this was imparted in a letter to one of the authors by L. Silberstein. As is well known, Levi-Civita and Weyl have given a general method for finding axially symmetric static solutions of the gravitational equations. By this method one can readily obtain a solution which, except for two point singularities lying on the axis of symmetry, is everywhere regular and is Euclidean at infinity. Hence if one admitted singularities as representing particles one would have here a case of two particles not accelerated by their gravitational interaction, which would certainly be excluded physically. Every field theory, in our opinion, must therefore adhere to the fundamental principle that singularities of the field are to be excluded. 
In the following we shall show that it is possible to do this in a natural way, that the question we are raising can be answered in the affirmative.

\section{\$1. A Special Kind of Singularity and Its REMOVAL}

The first step to the general theory of relativity was to be found in the so-called "Principle of Equivalence": If in a space free from gravitation a reference system is uniformly accelerated, the reference system can be treated as being "at rest," provided one interprets the condition of the space with respect to it as a homogeneous gravitational field. As is well known the latter is exactly described by the metric field ${ }^{1}$

$$
d s^{2}=-d x_{1}{ }^{2}-d x_{2}{ }^{2}-d x_{3}{ }^{2}+\alpha^{2} x_{1}{ }^{2} d x_{4}{ }^{2} .
$$

The $g_{\mu \nu}$ of this field satisfy in general the equations

$$
R^{i}{ }_{k l m}=0 \text {, }
$$

and hence the equations

$$
R_{k l}=R_{k l m}^{m}=0 .
$$

The $g_{\mu \nu}$ corresponding to (1) are regular for all finite points of space-time. Nevertheless one cannot assert that Eqs. (3) are satisfied by (1) for all finite values of $x_{1}, \cdots, x_{4}$. This is due to the fact that the determinant $g$ of the $g_{\mu \nu}$ vanishes for $x_{1}=0$. The contravariant $g^{\mu \nu}$ therefore become infinite and the tensors $R^{i}{ }_{k l m}$ and $R_{k l}$ take on the form $0 / 0$. From the standpoint of Eqs. (3) the hyperplane $x_{1}=0$ then represents a singularity of the field.

We now ask whether the field law of gravitation (and later on the field law of gravitation and electricity) could not be modified in a natural way without essential change so that the solution (1) would satisfy the field equations for all finite points, i.e., also for $x_{1}=0$. W. Mayer has called our attention to the fact that one can make $R^{i}{ }_{k l m}$ and $R_{k l}$ into rational functions of the $g_{\mu \nu}$ and their first two derivatives by multiplying them by suitable powers of $g$. It is easy to show

\footnotetext{
${ }^{1}$ It is worth pointing out that this metric field does not represent the whole Minkowski space but only part of it. Thus, the transformation that converts

into (1) is

$$
d s^{2}=-d \xi_{1}^{2}-d \xi_{2}{ }^{2}-d \xi_{3}{ }^{2}+d \xi_{4}{ }^{2}
$$$$
\begin{array}{ll}
\xi_{1}=x_{1} \cosh \alpha x_{4}, & \xi_{3}=x_{3}, \\
\xi_{2}=x_{2}, & \xi_{4}=x_{1} \sinh \alpha x_{4} .
\end{array}
$$

It follows that only those points for which $\xi_{1} 2 \geq \xi_{4}{ }^{2}$ correspond to points for which (1) is the metric.
}

that in $g^{2} R_{k l}$ there is no longer any denominator. If then we replace (3) by

$$
R_{k l} *=g^{2} R_{k l}=0
$$

this system of equations is satisfied by (1) at all finite points. This amounts to introducing in place of the $g^{\mu \nu}$ the cofactors $\left[g_{\mu \nu}\right]$ of the $g_{\mu \nu}$ in $g$ in order to avoid the occurrence of denominators. One is therefore operating with tensor densities of a suitable weight instead of with tensors. In this way one succeeds in avoiding singularities of that special kind which is characterized by the vanishing of $g$.

The solution (1) naturally has no deeper physical significance insofar as it extends into spatial infinity. It allows one to see however to what extent the regularization of the hypersurfaces $g=0$ leads to a theoretical representation of matter, regarded from the standpoint of the original theory. Thus, in the framework of the original theory one has the gravitational equations

$$
R_{i k}-\frac{1}{2} g_{i k} R=-T_{i k},
$$

where $T_{i k}$ is the tensor of mass or energy density. To interpret (1) in the framework of this theory we must approximate the line element by a slightly different one which avoids the singularity $g=0$. Accordingly we introduce a small constant $\sigma$ and let

$$
d s^{2}=-d x_{1}{ }^{2}-d x_{2}{ }^{2}-d x_{3}{ }^{2}+\left(\alpha^{2} x_{1}{ }^{2}+\sigma\right) d x_{4}{ }^{2} ;
$$

the smaller $\sigma(>0)$ is chosen, the nearer does this gravitational field come to that of (1). If one calculates from this the (fictitious) energy tensor $T_{i k}$ one obtains as nonvanishing components

$$
T_{22}=T_{23}=\alpha^{2} / \sigma /\left(1+\alpha^{2} x_{1}{ }^{2} / \sigma\right)^{2} .
$$

We see then that the smaller one takes $\sigma$ the more is the tensor concentrated in the neighborhood of the hypersurface $x_{1}=0$. From the standpoint of the original theory the solution (1) contains a singularity which corresponds to an energy or mass concentrated in the surface $x_{1}=0$; from the standpoint of the modified theory, however, (1) is a solution of (3a), free from singularities, which describes the "field-producing mass," without requiring for this the introduction of any new field quantities. 
It is clear that all equations of the absolute differential calculus can be written in a form free from denominators, whereby the tensors are replaced by tensor densities of suitable weight.

It is to be noted that in the case of the solution (1) the whole field consists of two equal halves, separated by the surface of symmetry $x_{1}=0$, such that for the corresponding points $\left(x_{1}, x_{2}, x_{3}\right.$, $\left.x_{4}\right)$ and $\left(-x_{1}, x_{2}, x_{3}, x_{4}\right)$ the $g_{i k}$ are equal. As a result we find that, although we are permitting the determinant $g$ to take on the value 0 (for $x_{1}=0$ ), no change of sign of $g$ and in general no change in the "inertial index" of the quadratic form (1) occurs. These features are of fundamental importance from the point of view of the physical interpretation, and will be encountered again in the solutions to be considered later.

\section{§2. The Schwarzschild Solution}

As is well known, Schwarzschild found the spherically symmetric static solution of the gravitational equations

$$
\begin{aligned}
& d s^{2}=-\frac{1}{1-2 m / r} d r^{2}-r^{2}\left(d \theta^{2}+\right.\left.\sin ^{2} \theta d \phi^{2}\right) \\
&+(1-2 m / r) d t^{2},
\end{aligned}
$$

( $r>2 m, \theta$ from 0 to $\pi, \phi$ from 0 to $2 \pi$ ); the variables $x_{1}, x_{2}, x_{3}, x_{4}$ are here $r, \theta, \phi, t$. The vanishing of the determinant of the $g_{\mu \nu}$ for $\theta=0$ is unimportant, since the corresponding (spatial) direction is not preferred. On the other hand $g_{11}$ for $r=2 m$ becomes infinite and hence we have there a singularity.

If one introduces in place of $r$ a new variable according to the equation

$$
u^{2}=r-2 m,
$$

one obtains for $d s^{2}$ the expression

$$
\begin{aligned}
d s^{2} & =-4\left(u^{2}+2 m\right) d u^{2} \\
& -\left(u^{2}+2 m\right)^{2}\left(d \theta^{2}+\sin ^{2} \theta d \phi^{2}\right)+\frac{u^{2}}{u^{2}+2 m} d t^{2} .
\end{aligned}
$$

These new $g_{\mu \nu}$ are regular functions for all values of the variables. For $u=0$, however, $g_{44}$ vanishes, hence also the determinant $g$. This does not prevent the field equations (3a), which have no denominators, from being satisfied for all values of the independent variables. We are therefore dealing with a solution of the (new) field equations, which is free from singularities for all finite points. The hypersurface $u=0$ (or in the original variables, $r=2 m$ ) plays here the same role as the hypersurface $x_{1}=0$ in the previous example.

As $u$ varies from $-\infty$ to $+\infty, r$ varies from $+\infty$ to $2 m$ and then again from $2 m$ to $+\infty$. If one tries to interpret the regular solution (5a) in the space of $r, \theta, \phi, t$, one arrives at the following conclusion. The four-dimensional space is described mathematically by two congruent parts or "sheets," corresponding to $u>0$ and $u<0$, which are joined by a hyperplane $r=2 m$ or $u=0$ in which $g$ vanishes. ${ }^{2}$ We call such a connection between the two sheets a "bridge."

We see now in the given solution, free from singularities, the mathematical representation of an elementary particle (neutron or neutrino). Characteristic of the theory we are presenting is the description of space by means of two sheets. A bridge, spatially finite, which connects these sheets characterizes the presence of an electrically neutral elementary particle. With this conception one not only obtains the representation of an elementary particle by using only the field equations, that is, without introducing new field quantities to describe the density of matter; one is also able to understand the atomistic character of matter as well as the fact that there can be no particles of negative mass. The latter is made clear by the following considerations. If we had started from a Schwarzschild solution with negative $m$, we should not have been able to make the solution regular by introducing a new variable $u$ instead of $r$; that is to say, no "bridge" is possible that corresponds to a particle of negative mass.

If we consider once more the solution (1) from the standpoint of the information we have acquired from the Schwarzschild solution, we see that there also the two congruent halves of the space for $x_{1}>0$ and $x_{1}<0$ can be interpreted as two sheets each corresponding to the same physical space. In this sense the example represents a gravitational field, independent of $x_{2}$ and $x_{3}$, which ends in a plane covered with mass and forming a boundary of the space. In this example, as well as in the Schwarzschild case, a solution free from singularities at all finite points is made possible by the introduction of the modified gravitational Eqs. (3a).

\footnotetext{
${ }^{2}$ Because of the symmetry about the hypersurface $g=0$, the sign of $g$ does not change at this hypersurface.
} 
The main value of the considerations we are presenting consists in that they point the way to a satisfactory treatment of gravitational mechanics. One of the imperfections of the original relativistic theory of gravitation was that as a field theory it was not complete; it introduced the independent postulate that the law of motion of a particle is given by the equation of the geodesic. ${ }^{3}$ A complete field theory knows only fields and not the concepts of particle and motion. For these must not exist independently of the field but are to be treated as part of it. On the basis of the description of a particle without singularity one has the possibility of a logically more satisfactory treatment of the combined problem: The problem of the field and that of motion coincide.

If several particles are present, this case corresponds to finding a solution without singularities of the modified Eqs. (3a), the solution representing a space with two congruent sheets connected by several discrete "bridges." Every such solution is at the same time a solution of the field problem and of the motion problem.

In this case it will not be possible to describe the whole field by means of a single coordinate system without introducing singularities. The simplest procedure appears to be to choose coordinate systems in the following way:

(1) One coordinate system to describe one of the congruent sheets. With respect to this system the field will appear to be singular at every bridge.

(2) One coordinate system for every bridge, to provide a description of the field at the bridge and in the neighborhood of the latter, which is free from singularities.

Between the coordinates of the sheet system and those of each bridge system there must exist outside of the hypersurfaces $g=0$, a regular coordinate transformation with nonvanishing determinant.

\section{§3. Combined Field. Electricity}

The simplest method of fitting electricity into the conceptual framework of the general theory of relativity is based on the following train of

3 To be sure, this weakness was formally avoided in the original theory of relativity by the introduction of the energy tensor into the field equations. It was clear, however, from the very beginning that this was' only a provisory completion of the theory in the sense of a phenomenological interpretation. thoughts. If besides the pure gravitational field other field variables are also present, the field equations of gravitation are

$$
R_{i k}-\frac{1}{2} g_{i k} R=-T_{i k},
$$

where $T_{i k}$ is the "material" energy tensor, i.e., that part of the mathematical expression of the energy which does not depend exclusively on the $g_{\mu \nu}$. In the case of the phenomenological representation of matter - if it is to be considered as "dust-like," that is, without pressure-one takes

$$
T^{i k}=\rho\left(d x^{i} / d s\right)\left(d x^{k} / d s\right),
$$

where $\rho$ is the density-scalar, $d x^{i} / d s$ the velocityvector of the matter. It is to be noted that $T_{4}{ }^{4}$ is accordingly a positive quantity.

In general the additional field-variables satisfy such differential equations that, in consequence of them, the divergence $T_{i k ; m} g^{k m}$ vanishes. As the divergence of the left side of (4) vanishes identically, this means that among all the field equations those four identities exist which are needed for their compatibility. Through this condition, in certain cases, the structure of $T_{i k}$, not however its sign, is determined. It appears natural to choose this sign in such a way that the component $T_{4}{ }^{4}$ (in the limit of the special relativity theory) is always positive.

The Maxwell electromagnetic field, as is well known, is represented by the antisymmetric field tensor $\varphi_{\mu \nu}\left(=\partial \varphi_{\mu} / \partial x^{\nu}-\partial \varphi_{\nu} / \partial x^{\mu}\right)$, which satisfies the field equations

$$
\varphi_{\mu \nu ; \sigma} g^{\nu \sigma}=0 .
$$

These equations have the well-known consequence that the divergence of the tensor

$$
T_{i k}=\frac{1}{4} g_{i k} \varphi_{\alpha \beta} \varphi^{\alpha \beta}-\varphi_{i \alpha} \varphi_{k}{ }^{\alpha}
$$

vanishes. The sign has been so chosen that $T_{4}^{4}$ is positive for the case of the special relativity theory. If one puts this $T_{i k}$ into the gravitational Eqs. (4), then the latter together with (6) and (7) form a theory of gravitation and electricity.

It so happens that we are forced to put the negative of the above into the gravitational equations if it is to be possible to obtain static spherically symmetric solutions of the equations, free from singularities, which could represent electrical particles. Making this change of sign one finds as the required solution 


$$
\left.\begin{array}{c}
\varphi_{1}=\varphi_{2}=\varphi_{3}=0, \quad \varphi_{4}=\epsilon / r \\
d s^{2}=-\frac{1}{1-2 m / r-\epsilon^{2} / 2 r^{2}} d r^{2} \\
-r^{2}\left(d \theta^{2}+\sin ^{2} \theta d \varphi^{2}\right)+\left(1-\frac{2 m}{r}-\frac{\epsilon^{2}}{2 r^{2}}\right) d t^{2} .
\end{array}\right\}
$$

Here $m$ has obviously the significance of a gravitating mass, $\epsilon$ that of an electrical charge.

It turns out that also in this case there is no difficulty in forming a solution without singularity corresponding to the solution just given. ${ }^{4}$ Curiously enough, one finds that the mass $m$ is not determined by the electrical charge $\epsilon$, but that $\epsilon$ and $m$ are independent constants of integration. It also turns out that for the removal of the singularity it is not necessary to take the ponderable mass $m$ positive. In fact, as we.shall show immediately, there exists a solution free from singularities for which the mass constant $m$ vanishes. Because we believe that these massless solutions are the physically important ones we will consider here the case $m=0$.

The field equations without denominators can be written

$$
\begin{aligned}
\varphi_{\mu \nu}=\varphi_{\mu, \nu}-\varphi_{\nu, \mu}, \quad g^{2} \varphi_{\mu \nu ; \sigma} g^{\nu \sigma}=0, \\
g^{2}\left(R_{i k}+\varphi_{i \alpha} \varphi_{k}{ }^{\alpha}-\frac{1}{4} g_{i k} \varphi_{\alpha \beta} \varphi^{\alpha \beta}\right)=0,
\end{aligned}
$$

where in the last equation the term in $R$ has been omitted because it vanishes in consequence of (7), by which $T_{\alpha}{ }^{\alpha}$ is zero.

If in Eq. (8) (with $m=0$ ) one replaces $r$ by the variable $u$ according to the equation

one obtains

$$
u^{2}=r^{2}-\epsilon^{2} / 2
$$

$$
\left.\begin{array}{c}
\varphi_{1}=\varphi_{2}=\varphi_{3}=0, \quad \varphi_{4}=\epsilon /\left(u^{2}+\epsilon^{2} / 2\right)^{\frac{1}{2}} \\
d s^{2}=-d u^{2}-\left(u^{2}+\epsilon^{2} / 2\right)\left(d \theta^{2}+\sin ^{2} \theta d \phi^{2}\right) \\
+\left[2 u^{2} /\left(2 u^{2}+\epsilon^{2}\right)\right] d t^{2} .
\end{array}\right\}
$$

This solution is free from singularities for all finite points in the space of two sheets and the charge is again represented by a bridge between the sheets. It is the representation of an elementary electrical particle without mass.

\footnotetext{
${ }^{4}$ If we had taken the usual sign for $T_{i k}$, the solution would involve $+\epsilon^{2}$ instead of $-\epsilon^{2}$. It would then not be possible, by making a coordinate transformation, to obtain a solution free from singularities.
}

\section{\$4. Summary and General Remarks}

If one solves the equations of the general theory of relativity for the static spherically symmetric case, with or without an electrostatic field, one finds that singularities occur in the solutions. If one modifies the equations in an unessential manner so as to make them free from denominators, regular solutions can be obtained, provided one treats the physical space as consisting of two congruent sheets. The neutral, as well as the electrical, particle is a portion of space connecting the two sheets (bridge). In the hypersurfaces of contact of the two sheets the determinant of the $g_{\mu \nu}$ vanishes.

One might expect that processes in which several elementary particles take part correspond to regular solutions of the field equations with several bridges between the two equivalent sheets corresponding to the physical space. Only by investigations of these solutions will one be able to determine the extent to which the theory accounts for the facts. For the present one cannot even know whether regular solutions with more than one bridge exist at all.

It appears that the most natural electrical particle in the theory is one without gravitating mass. One is therefore led, according to this theory, to consider the electron or proton as a two-bridge problem.

In favor of the theory one can say that it explains the atomistic character of matter as well as the circumstance that there exist no negative neutral masses, that it introduces no new variables other than the $g_{\mu \nu}$ and $\varphi_{\mu}$, and that in principle it can claim to be complete (or closed). On the other hand one does not see $a$ priori whether the theory contains the quantum phenomena. Nevertheless one should not exclude $a$ priori the possibility that the theory may contain them. Thus it might turn out that only such regular many-bridge solutions can exist for which the "charges" of the electrical bridges are numerically equal to one another and only two different "masses" occur for the mass bridges, and for which the stationary "motions" are subject to restrictions like those which we encounter in the quantum theory.

In any case here is a possibility for a general relativistic theory of matter which is logically completely satisfying and which contains no new hypothetical elements. 